\title{
Association between thyroid-stimulating hormone and maternal hemodynamics in hypertensive disorders of pregnancy: an observational study
}

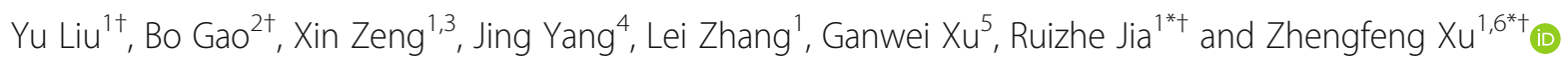

\begin{abstract}
Background: Hypertensive disorders of pregnancy (HDP) are characterized by hemodynamic disturbances. Altered thyroid function is a risk factor for poor outcomes of pregnancy. However, the associations between thyroid function biomarkers and maternal hemodynamics during pregnancy in HDP remain unclear.

Methods: From January 2016 to January 2018, pregnant women diagnosed with HDP admitted to the Nanjing Maternity and Child Health Care Hospital were prospectively enrolled in the third trimester. Normally distributed variables were expressed as mean \pm standard deviation and skewed variables were expressed as median (25th percentile, 75th percentile). Correlations between thyroid-stimulating hormone (TSH) or free thyroxine (FT4) and maternal hemodynamic parameters were assessed by Pearson's correlation coefficient and 95\% confidence interval $(95 \% \mathrm{Cl})$. Bonferroni's correction for multiple correlations was performed. Logistic regression models with odd ratio (OR) and $95 \% \mathrm{Cl}$ were applied to confirm the associations.
\end{abstract}

Results: A total of 163 third-trimester pregnant women with HDP with a mean gestational age of $35.62 \pm 2.83$ weeks were recruited. The infant birth weight of patients with elevated TSH levels was lower than that of patients with normal TSH levels ( $2635 \pm 867 \mathrm{~g}$ vs. $3037 \pm 673 \mathrm{~g}, p=0.002)$. Reduced cardiac output (CO) was defined as $\mathrm{CO}<$ $3.5 \mathrm{~L} / \mathrm{min}$. The infant birth weight of patients with reduced $\mathrm{CO}$ was lower than that of patients with normal CO ( $2250 \pm 510 \mathrm{~g}$ vs. $2890 \pm 774 \mathrm{~g}, p=0.002)$. TSH levels were significantly and negatively correlated with $\mathrm{CO}(\mathrm{r}=-$ $0.260,95 \% \mathrm{Cl}:-0.392--0.103, p<0.001)$. FT4 levels were not significantly correlated with any of the maternal hemodynamic parameters (all $p>0.05)$. TSH level $(\mathrm{OR}=1.371,95 \% \mathrm{Cl}: 1.086-1.733, p=0.008)$ was confirmed associated with reduced $\mathrm{CO}$ in the logistic regression analysis.

Conclusions: Elevated TSH levels are associated with reduced CO in HDP during the third trimester.

Keywords: Hypertensive disorders of pregnancy, Thyroid stimulating hormone, Maternal hemodynamics, Cardiac output, Systemic vascular resistance

\footnotetext{
*Correspondence: rzjia9599@163.com; xzfnify@163.com

†Yu Liu and, Bo Gao contributed equally as co-first authors, Ruizhe Jia and Zhengfeng Xu contributed equally to this work as co-corresponding authors. 'Department of Obstetrics and Gynecology, The affiliated Obstetrics and Gynecology Hospital with Nanjing Medical University; Nanjing Maternity and Child Health Care Hospital, 123 Tianfeixiang, Mochou Road, Qinhuai District, Nanjing 210004, China

Full list of author information is available at the end of the article
}

(c) The Author(s). 2019 Open Access This article is distributed under the terms of the Creative Commons Attribution 4.0 International License (http://creativecommons.org/licenses/by/4.0/), which permits unrestricted use, distribution, and reproduction in any medium, provided you give appropriate credit to the original author(s) and the source, provide a link to the Creative Commons license, and indicate if changes were made. The Creative Commons Public Domain Dedication waiver (http://creativecommons.org/publicdomain/zero/1.0/) applies to the data made available in this article, unless otherwise stated. 


\section{Background}

Hypertensive disorders of pregnancy (HDP) are common complications during pregnancy, with incidence rates of approximately $2-8 \%[1,2]$, and can negatively affect gestational outcomes [1]. At an advanced and more severe stage, preeclampsia (PE), which is characterized by gestational hypertension and nephrotic impairment with proteinuria, is one of the most deleterious gestational hypertensive disorders, with high mortality during pregnancy $[3,4]$. Gestational hypertension and PE have similar symptoms and can both lead to intrauterine fetal growth restriction (FGR), still birth, preterm delivery, placental abruption and disseminated intravascular coagulation [3], which seriously threaten the health of both the mother and fetus. However, the exact etiology of HDP, as well as effective prophylaxis and therapeutic targets, remain unclarified [3-6]. The commonly accepted pathogenesis of HDP is the abnormal reconstruction of the spiral artery and placental ischemia [7]. Inflammatory factors secondary to placental ischemia also play a key role in inducing vasoconstriction dysfunction in HDP [5]. Moreover, studies have suggested that obesity and thyroid dysfunction are major risk factors for the development of HDP $[8,9]$.

Maternal hemodynamic alterations occur in pregnant women with HDP $[10,11]$. As part of the process of adaptation to pregnancy, cardiac output (CO) tends to increase, and systemic vascular resistance (SVR) tends to decrease in normotensive women [12]. In contrast, women with HDP fail to undergo this hemodynamic adaptation, with a consequent and significant increase in SVR [10-12]. By conducting a longitudinal study, Ghi et al. postulated that hemodynamic disturbances may occur even in the early stages of pregnancy and can anticipate the development of pregnancy-induced hypertension [13]. Tay et al. discovered that a poor prognosis of HDP, especially in terms of FGR, is significantly associated with declining CO [14]. Peripheral vasoconstriction secondary to maternal systemic inflammation and endothelial cell activation are also considered to be responsible for hypertensive disorders during pregnancy [3]. However, the risk factors for the occurrence of these pathophysiological hemodynamic changes have not been fully investigated.

Thyroid dysfunction frequently occurs during pregnancy [1]. Both hyper- and hypothyroidism can negatively affect the prognosis of pregnancy. Moreover, thyroid dysfunction is considered to be correlated with gestational hypertensive disorders $[15,16]$. Wilson et al. discovered that the severity of PE is positively correlated with the level of thyroid-stimulating hormone (TSH). By multivariate analysis, hypothyroidism has been confirmed to be an independent risk factor for severe PE and fetal growth restriction [15-17]. However, the underlying roles of thyroid function in hypertensive disorders during pregnancy are still poorly established. In the general population, thyroid function affects the cardiovascular system [18]. However, the associations between thyroid function and maternal hemodynamics during pregnancy in HDP remained uninvestigated.

\section{Materials and methods Patients}

From January 2016 to January 2018, pregnant women with HDP who were admitted to the Nanjing Maternity and Child Health Care Hospital were prospectively enrolled in the third trimester. Data were analyzed retrospectively from our prospectively collected dataset. The diagnostic criterion for HDP was an increase in blood pressure to $\geq 140 / 90 \mathrm{mmHg}$ after 20 gestational weeks in previously normotensive women [19]. The elevated blood pressure was confirmed by at least 2 measurements at intervals of at least $4-6 \mathrm{~h}$ apart. The exclusion criteria were patients with multiple pregnancies, patients with a history of cardiac disorders or chronic hypertension, patients diagnosed with chronic hypertension in pregnancy, preeclampsia superimposed on chronic hypertension, patients with a history of renal dysfunction, patients with a history of thyroid disorders, patients taking thyroxine substitution medicines before hemodynamic exams, patients taking anti-hypertensive drugs before hemodynamic exams, patients with a history of in-vitro fertilization treatment, patients who consumed alcohol or smoked, patients with other chronic diseases and patients lacking complete medical records. With accordance to the principles of the Declaration of Helsinki, this observational study was approved by the Clinical Research Ethics Committee of the Nanjing Maternity and Child Health Care Hospital. Written consent was obtained from all patients enrolled.

\section{Maternal hemodynamic monitoring}

We applied a noninvasive hemodynamic monitoring technique with electronic impedance cardiography (BioZ CardioDynamics, San Diego, USA) to analyze the circulatory function of HDP patients at enrollment. Electronic impedance cardiography is widely used and enables clinicians to quickly and easily observe the cardiac function and peripheral resistance of patients. The performance of electronic impedance cardiography in pregnancy was validated previously [20], and the protocols of electronic impedance cardiography monitoring have been described [20]. Briefly, patients were asked to remain calm and rest for $30 \mathrm{~min}$ before electronic impedance cardiography monitoring and were scanned in the left lateral decubitus position. The measurements of all patients were performed by one experienced sonographer. The recorded hemodynamic parameters included systolic blood pressure, diastolic blood pressure, mean arterial 
pressure, CO, stroke volume, SVR, ejection time percent, peak velocity of flow, mean pressure gradient and velocity time integral. In compliance with the instructions of the electronic impedance cardiography monitor for pregnancy applied in the present study, low $\mathrm{CO}$ was defined as $\mathrm{CO}<3.5 \mathrm{~L} / \mathrm{min}$, and high $\mathrm{SVR}$ was defined as SVR $>1600$ dyne $\times \mathrm{sec} / \mathrm{cm}^{5}$.

\section{Thyroid function measurements}

Fasting maternal venous blood samples were obtained on the day of electronic impedance cardiography monitoring. Samples were centrifuged, and supernatants were stored at $-80^{\circ} \mathrm{C}$. TSH was measured using an Elecsys-TSH kit (Roche Diagnostics, Mannheim, Germany, RRID:AB_ 2756377). FT4 was measured using an FT4 Flex reagent (Siemens, Berlin, Germany, RRID:AB_2801666). Thyroid peroxidase antibody was measured with an E-Anti-TPO kit (Roche Diagnostics, Mannheim, Germany, RRID:AB_ 10698637) and was graded as positive when greater than $60 \mathrm{IU} / \mathrm{ml}$. The normal reference for TSH in the third trimester was 0.3 to $3.5 \mathrm{mIU} / \mathrm{L}$, as recommended [21].

\section{Covariates}

The demographic information of the patients included age, body mass index (BMI, defined as body weight in kilograms/ square of the body height in meters) before pregnancy, gravidity, parity, gestational age at enrollment and complications of PE. The diagnostic criterion for PE was the presence of HDP with urinary protein above $300 \mathrm{mg} /$ $24 \mathrm{~h}$ [19]. Laboratory parameters were measured from fasting venous blood samples on the day of electronic impedance cardiography monitoring. The laboratory parameters included the urea nitrogen level, creatinine level, glomerular filtration rate (GFR) and albumin level. The 24-h urine protein levels were also recorded.

\section{Statistical analysis}

Data were analyzed by SPSS version 20.0 (IBM, Chicago, USA, RRID:SCR_002865) and MedCalc Statistical Software version 15.2.2 (MedCalc Software bvba, Ostend, Belgium, RRID:SCR_015044). Normally distributed continuous variables were expressed as the means \pm standard deviations, skewed data were expressed as medians (25th percentile,75th percentile), and the data were analyzed by independent Student's t-test and the Mann-Whitney $\mathrm{U}$ test, respectively. Categorical variables were expressed as frequencies and proportions, and they were analyzed with the chi-square test ( $x^{2}$-test). Correlations between thyroid function biomarkers and maternal hemodynamic parameters were assessed with Pearson's correlation coefficient and 95\% confidence interval (95\%CI). Bonferroni's correction for multiple correlations was performed. The maternal hemodynamic parameters that had significant correlations with thyroid function biomarkers per
Pearson's correlation coefficient were stratified for logistic regression, and thyroid function biomarkers were plotted into univariate and multivariate regression models (per the stepwise method) with odd ratio (OR) and $95 \% \mathrm{CI}$ to confirm the associations. A two-sided $p<$ 0.05 was considered statistically significant.

\section{Results}

\section{Baseline characteristics}

The present study consisted of 163 pregnant women with a clear diagnosis of HDP. All patients were enrolled in the third trimester with a mean gestational age of $35.62 \pm 2.83$ weeks. The mean levels of TSH and FT4 were $3.67 \pm 2.21$ $\mathrm{mIU} / \mathrm{L}$ and $11.58 \pm 2.15 \mathrm{pmol} / \mathrm{L}$, respectively (Table 1 ). Regarding the maternal hemodynamics, the mean level of $\mathrm{CO}$ was $5.66 \pm 1.43 \mathrm{~L} / \mathrm{min}$. The proportion of pregnant

Table 1 Baseline characteristics

\begin{tabular}{|c|c|}
\hline Characteristic & $N=163$ \\
\hline Age (year) & $30.83 \pm 5.06$ \\
\hline $\mathrm{BMI}\left(\mathrm{kg} / \mathrm{m}^{2}\right)$ & $21.94 \pm 4.14$ \\
\hline \multicolumn{2}{|l|}{ Gravidity (n, \%) } \\
\hline 1 & $104(63.8)$ \\
\hline 2 & $43(26.4)$ \\
\hline$\geq 3$ & $16(9.8)$ \\
\hline \multicolumn{2}{|l|}{ Parity (n, \%) } \\
\hline 0 & $109(66.8)$ \\
\hline 1 & $50(30.7)$ \\
\hline$\geq 2$ & $4(2.5)$ \\
\hline Gestational age at enrollment (weeks) & $35.62 \pm 2.83$ \\
\hline \multicolumn{2}{|l|}{ HDP } \\
\hline Gestational hypertension & $86(52.8)$ \\
\hline Preeclampsia & $77(47.2)$ \\
\hline \multicolumn{2}{|l|}{ Laboratory } \\
\hline Urea nitrogen (mmol/L) & $4.36 \pm 1.81$ \\
\hline Creatinine (umol/L) & $56.17 \pm 22.57$ \\
\hline GFR $\left(\mathrm{ml} / \mathrm{min} / 1.73 \mathrm{~m}^{2}\right)$ & $132.19 \pm 44.77$ \\
\hline Albumin (g/L) & $32.02 \pm 5.65$ \\
\hline Urinary protein (mg/24 h) & $211(75,820)$ \\
\hline \multicolumn{2}{|l|}{ Thyroid function } \\
\hline $\mathrm{TSH}(\mathrm{mlU} / \mathrm{L})$ & $3.67 \pm 2.21$ \\
\hline FT4 (pmol/L) & $11.58 \pm 2.15$ \\
\hline Thyroid peroxidase antibody positive (n, \%) & $6(3.7)$ \\
\hline Birth weight (g) & $2862.07 \pm 787.48$ \\
\hline Birth weight $<2500 \mathrm{~g}(\mathrm{n}, \%)$ & $49(30.1)$ \\
\hline Gestational age at delivery (weeks) & $37.49 \pm 2.88$ \\
\hline
\end{tabular}

BMI: body mass index, HDP: hypertensive disorders of pregnancy, GFR: glomerular filtration rate, TSH: for thyroid stimulating hormone, FT4: for free thyroxine. The values of urinary protein were not normally distributed and were expressed as median (25th percentile, 75th percentile), other parameters were normally distributed and were expressed as mean \pm standard deviations 
women with HDP who had CO reductions was 27.6\% (Table 2). Patients with reduced CO (Additional file 1: Table S1) had higher rates of low-birth-weight infants ( $44.4 \%$ vs. $24.6 \%, p=0.013$ ). There were 71 patients with TSH levels above $3.5 \mathrm{mIU} / \mathrm{L}$. The birth weights of the infants of the patients with normal TSH levels and elevated TSH levels were $3037 \pm 673 \mathrm{~g}$ and $2635 \pm 867 \mathrm{~g}$, respectively $(p=0.002)$ and the proportions of low-birth-weight infants were 15.2 and $49.3 \%$, respectively $(p<0.001)$.

\section{Pearson's correlations between thyroid function} biomarkers and maternal hemodynamic parameters

The TSH level was significantly and negatively correlated with CO ( $\mathrm{r}=-0.260, \mathrm{p}<0.001,95 \% \mathrm{CI}$ : $-0.392--0.103)$, indicating that cardiac output decreased with increasing TSH levels. The TSH level was significantly and positively correlated with SVR $(\mathrm{r}=0.162$, 95\%CI: 0.009-0.308, $p=$ 0.039), indicating that systemic vascular resistance increased with increasing TSH levels. After adjustments for multiple correlations by Bonferroni's correction, only $\mathrm{CO}$ was significantly correlated with TSH. The TSH level was not correlated with other maternal hemodynamic parameters (all $p>0.05$ ). The FT4 level was not significantly correlated with any of the maternal hemodynamic parameters (all $\mathrm{p}>0.05$ ). The $95 \% \mathrm{CI}$ for the $\mathrm{r}$ values were shown in Table 3.

\section{Univariate and multivariate logistic regression models for reduced $\mathrm{CO}$}

According to the electronic impedance cardiography machine instructions for pregnancy, women with $\mathrm{CO}<3.5 \mathrm{~L} /$ min were categorized as the $\mathrm{CO}$ reduction group. BMI before pregnancy $(p=0.046)$, complications of $\operatorname{PE}(p=$ $0.044)$, GFR $(p=0.049)$, albumin level $(p=0.033)$ and TSH

Table 2 Maternal hemodynamic monitoring of the study population

\begin{tabular}{ll}
\hline Hemodynamic parameters & $\mathrm{N}=163$ \\
\hline Systolic blood pressure $(\mathrm{mmHg})$ & $148.13 \pm 18.54$ \\
Diastolic blood pressure $(\mathrm{mmHg})$ & $94.49 \pm 12.27$ \\
Mean arterial pressure $(\mathrm{mmHg})$ & $112.47 \pm 13.73$ \\
$\mathrm{CO}(\mathrm{L} / \mathrm{min})$ & $5.66 \pm 1.43$ \\
Stroke volume $(\mathrm{ml} / \mathrm{kg})$ & $0.92 \pm 0.23$ \\
Ejection time percent $(\%)$ & $47.39 \pm 5.96$ \\
SVR (dynexsec/cm $\left.{ }^{5}\right)$ & $1632.85 \pm 449.04$ \\
Peak velocity of flow (m/s) & $1.2(1.00,1.40)$ \\
Mean pressure gradient & $2.3(1.90,3.20)$ \\
Velocity time integral & $25(22,28)$ \\
\hline
\end{tabular}

CO: cardiac output; SVR: systemic vascular resistance. Peak velocity of flow, mean pressure gradient and velocity time integral were skewed distributed and were expressed as median (25th percentile, 75 th percentile), other parameters were normally distributed and were expressed as mean \pm standard deviations level $(p<0.001)$ were significantly different between the $\mathrm{CO}$ normal and $\mathrm{CO}$ reduction groups (Additional file 1: Table S1). Therefore, they were considered as confounding factors in the adjusted model and were pooled into the univariate regression. Parameters with $p$-values less than 0.05 in the univariate regression were adjusted in the multivariate analysis. After adjustments for confounders, the TSH level $(\mathrm{OR}=1.371$ 95\%CI: 1.086-1.733, $p=0.008)$ was confirmed to be significantly associated with $\mathrm{CO}$ reduction. (Table 4).

This observation demonstrated that elevated TSH levels were associated with reduced cardiac output in pregnant women with HDP in the third trimester.

\section{Discussion}

Previous findings suggested that both overt and subclinical hypothyroidism during pregnancy are associated with a negative gestational prognosis, including the occurrence of gestation induced hypertension and $\mathrm{PE}$, spontaneous abortion, premature delivery, fetal distress, fetal growth restriction and fetal death [15, 16, 22, 23]. A meta-analysis of hypothyroidism in pregnancy showed a 2.4-fold and 1.78-fold increased risk for fetal growth restriction and low birth weight, respectively [22]. However, the underlying mechanisms of this pathogenesis have not been clearly established. Barjaktarovic et al. indicated that thyroid function was associated with placental vascular function and placental hemodynamics during the second and third trimesters [9]. Vsilopoulou et al. suggested that thyroid function hormones play a role in trophoblast cell invasion and placentation [17]. Liu et al. postulated that thyroid function is essential in metabolism and protein synthesis, as well as in tissue differentiation and maturation [22, 24]. Researchers have suggested that even with normal FT4 levels, women with increased TSH levels during pregnancy need higher levels of thyroid hormone to ensure fetal development [25]. Our research demonstrated that patients with elevated TSH levels, which may include those with hypothyroidism during pregnancy, had significantly higher rates of low-birth-weight infants. Moreover, our study also found that the proportion of low-birth-weight infants was also higher in patients with hemodynamic disturbances. Perinatal outcomes were poorer for both women with higher TSH levels and those with reduced $\mathrm{CO}$, which provides clues regarding the potential linkage between TSH and maternal hemodynamic alterations.

Thyroid dysfunction is closely correlated with cardiovascular disorders in the general population [26, 27]. Li et al. conducted a study of 184 patients with nonischemic dilated cardiomyopathy and discovered an association between TSH level and poor cardiac prognosis. Thyroid hormones regulate beta-adrenergic positive chronotropic effects, which may lead to a hyperdynamic state and increase cardiac preload [28]. Hypothyroidism-related systemic vascular 
Table 3 Pearson correlation of thyroid function indicators with hemodynamic parameters in overall gestational hypertension patients

\begin{tabular}{|c|c|c|c|c|c|c|}
\hline \multirow[t]{2}{*}{$\mathrm{N}=163$} & \multicolumn{3}{|l|}{ TSH } & \multicolumn{3}{|l|}{ FT4 } \\
\hline & r & 95\%Cl (lower, upper) & $\mathrm{p}$ & r & 95\%Cl (lower, upper) & $p$ \\
\hline Systolic blood pressure & 0.132 & $-0.022,0.280$ & 0.094 & -0.056 & $-0.208,0.099$ & 0.481 \\
\hline Diastolic blood pressure & 0.144 & $-0.010,0.291$ & 0.067 & 0.002 & $-0.151,0.156$ & 0.976 \\
\hline Mean arterial pressure & 0.117 & $-0.037,0.266$ & 0.136 & -0.062 & $-0.213,0.093$ & 0.435 \\
\hline Peak velocity of flow & 0.016 & $-0.138,0.170$ & 0.836 & -0.097 & $-0.247,0.057$ & 0.217 \\
\hline Mean pressure gradient & -0.027 & $-0.180,0.128$ & 0.736 & -0.108 & $-0.258,0.046$ & 0.169 \\
\hline Velocity time integral & 0.018 & $-0.136,0.172$ & 0.816 & -0.155 & $-0.302,-0.002$ & 0.048 \\
\hline $\mathrm{CO}$ & -0.260 & $-0.392,-0.103$ & $0.001^{* * *}$ & -0.072 & $-0.228,0.077$ & 0.361 \\
\hline Stroke volume & 0.022 & $-0.132,0.175$ & 0.779 & -0.111 & $-0.260,0.044$ & 0.159 \\
\hline Ejection time percent (\%) & -0.095 & $-0.245,0.060$ & 0.227 & -0.015 & $-0.169,0.139$ & 0.846 \\
\hline SVR & 0.162 & $0.009,0.308$ & 0.039 & 0.044 & $-0.111,0.196$ & 0.581 \\
\hline
\end{tabular}

Remarks: *** indicated for $p<0.001 .95 \% \mathrm{Cl}$ : $95 \%$ confidence interval. CO: cardiac output; SVR: systemic vascular resistance. Bonferroni's correction for multiple correlations: $p<0.0025$

resistance and endothelial dysfunction may play a pivotal role in the negative prognosis of cardiovascular diseases [29]. Biondi et al. [30] also suggested that hypothyroidism correlated with increased cardiovascular morbidity and mortality due to its negative effect on cardiac contractility, systemic vascular resistance, and endothelial function. Previous studies have postulated that hypothyroidism, even in the subclinical stage, could change cardiac function by altering sarcoplasmic reticulum calcium-ATPase and the transcriptions of other gene products to affect myocyte contractility and dilation [31]. In addition, the elevation in TSH levels could inhibit the synthesis of the endothelial vasodilators, thereby leading to arterial stiffness [31]. Cardiac output may be reduced by approximately $30-50 \%$ in the presence of hypothyroidism, as previously reported [32]. Roef et al. conducted a population-based study and found that alterations in thyroid hormone levels, even within the normal range, contributed to alterations in heart rate and the reconstruction of cardiac structure [33]. Systolic blood pressure was also confirmed to be positively associated with TSH [33]. By establishing animal models, Gao et al. demonstrated a positive correlation between the TSH level and contractility index, endothelin. A negative correlation was found with heart rate, systolic blood pressure, the maximal rate of the pressure rise and left ventricular systolic pressure [34]. All of the above studies were based on the general population. The relationship between TSH level and cardiovascular function was also confirmed in new-born infants with congenital hypothyroidism [35]. However, to date, there is no report on the association between TSH level and maternal cardiovascular function during pregnancy. Our research demonstrated that in women with HDP, TSH level is positively correlated with reduced $\mathrm{CO}$, which supports the findings of previous studies.

In the present study, TSH levels were significantly and negatively correlated with $\mathrm{CO}$. These results indicated that cardiac output in HDP declined with the elevation in serum TSH during the third trimester. The physiological adaptation of the cardiovascular system in normal pregnancy was characterized by a significant increase in CO [12, 36], which enables maternal compliance with the increased metabolic demands for fetal growth [37]. The previously reported mean percent increase in $\mathrm{CO}$ ranges from 13 to $45 \%$ during the first trimester [36]. HDP,

Table 4 Association between thyroid function indicators and reduced CO

\begin{tabular}{|c|c|c|c|c|}
\hline & \multicolumn{2}{|l|}{ Univariate } & \multicolumn{2}{|l|}{ Multivariate } \\
\hline & OR $(95 \% \mathrm{Cl})$ & $p$ & OR $(95 \% \mathrm{Cl})$ & $p$ \\
\hline $\mathrm{BMI}$ & $1.140(1.014-1.281)$ & $0.034^{*}$ & $1.161(1.026-1.314)$ & $0.018^{*}$ \\
\hline GFR & $0.983(0.968-0.999)$ & $0.032^{*}$ & $0.987(0.970-1.004)$ & 0.141 \\
\hline Albumin & $0.934(0.834-1.046)$ & 0.237 & & \\
\hline $\mathrm{TSH}$ & $1.397(1.126-1.733)$ & $0.002^{* *}$ & $1.371(1.086-1.733)$ & $0.008^{* *}$ \\
\hline Complications of PE & $1.092(0.320-3.721)$ & 0.889 & & \\
\hline
\end{tabular}

Remarks: * indicated for $\mathrm{p}<0.05$; ${ }^{*}$ indicated for $p<0.01$. In the study population, 45 pregnant women were with low CO and the remained 118 patients were with normal CO level. Baseline factors with $p$ value less than 0.05 in comparison between normal CO and low CO groups (Additional file 1: Table S1) were pooled into univariate logistic regression model. Factors with $\mathrm{p}$-value less than 0.05 in univariate regression were pooled into multivariate logistic regression analysis 
as frequent complications during pregnancy, is a leading cause of maternal and neonatal mortality and morbidity [38]. Maternal hemodynamic alteration of patients with HDP was considered as rising in SVR secondary to inflammatory factors induced vasoconstriction dysfunction [38]. Maternal hemodynamic disturbances secondary to inadaptation to normal pregnancy play an essential role in adverse perinatal outcomes and postpartum complications [14]. Although the trends regarding $\mathrm{CO}$ alterations in HDP are conflicting between different studies, fetal growth restriction was demonstrated to be associated with inadequate CO [14, 37-39]. One indicated that decreased TSH levels were associated with increased $\mathrm{CO}$ and an increased risk of pulmonary hypertension in a general population of females [40]. However, this investigation in the context of pregnancy was not conducted. To our knowledge, this is the first study on the association between TSH and maternal hemodynamics in HDP during pregnancy. The co-existing correlation between elevated TSH and reduced $\mathrm{CO}$ may provide new insight into the underlying mechanism of the linkage between hypothyroidism in pregnancy and FGR or low birth weight in HDP patients.

There were several limitations in the present research. First, this study contained a small sample size and was based on a single-center design assessing a Chinese population. Multi-center investigations containing larger sample sizes should be conducted further. Second, the patients in the present study were enrolled during the third trimester, and the conclusions are limited to this trimester. Studies on longitudinal changes in TSH and hemodynamics are warranted in the future. Third, this study lacked a comparison with normotensive pregnancies. It was unclear whether this association existed regardless of whether a woman had a hypertensive pregnancy.

\section{Conclusions}

The research revealed that pregnancies with elevated TSH levels and reduced $\mathrm{CO}$ have higher rates of lowbirth-weight infants. Elevated TSH levels were associated with reduced $\mathrm{CO}$ in HDP women during the third trimester of pregnancy. The co-existing correlation between elevated TSH level and reduced $\mathrm{CO}$ may provide new insight into the underlying mechanism of the linkage between hypothyroidism in pregnancy and FGR or low birth weight in HDP patients. Mechanisms of the associations between TSH and reduced $\mathrm{CO}$ warrants future investigation.

\section{Supplementary information}

Supplementary information accompanies this paper at https://doi.org/10. 1186/s12884-019-2556-4.

Additional file 1: Table S1. Comparison of baseline characteristics between normal $\mathrm{CO}$ and reduced $\mathrm{CO}$ groups.

\section{Abbreviations}

95\%Cl: 95\% confidence interval; BMl: body mass index; CO: cardiac output; FGR: fetal growth restriction; FT4: free thyroxine; GFR: glomerular filtration rate; HDP: hypertensive disorders of pregnancy; OR: odd ratio;

PE: preeclampsia; SD: standard deviation; SVR: systemic vascular resistance; TSH: thyroid stimulating hormone

\section{Acknowledgements}

We acknowledge all the women with HDP participated in the study. We are also grateful to all the staff who have helped us in the study. Bo Gao and Yu Liu contributed equally to this article as co-first authors. Zhengfeng $\mathrm{Xu}$ and Ruizhe Jia contributed equally to this work as co-corresponding authors.

\section{Authors' contributions}

YL helped in study design, data collection, manuscript writing; BG collected data and wrote the manuscript; XZ helped in data interpretation and data analysis; JY performed data analysis; LZ helped in figure editing, data analysis; GW Xu collected data; RZ Jia helped in study design, financial support; ZF Xu helped in study design, manuscript supervision. All authors have read and approved the final manuscript, and they take responsibility for the authenticity of the research.

\section{Funding}

This study was supported by grants from the National Natural Science Foundation of China (No. 81571444). The funding was used for providing clinical examinations for patients enrolled. Nanjing Medical Science and Technology Development Foundation (No. YKK15163) was used for interpreting data and statistical analysis. The Project of Invigorating Health Care through Science, Technology and Education-the Jiangsu Provincial Medical Youth Talent was used for data collection and language editing service.

\section{Availability of data and materials \\ The datasets used during the current study are available from the} corresponding author on reasonable request.

\section{Ethics approval and consent to participate}

The study was in accordance with the principles of the Declaration of Helsinki and was approved by the Clinical Research Ethics Committee of the Nanjing Maternity and Child Health Care Hospital. This research involved blood samples of human participants and written informed consent was obtained from all participants.

Consent for publication Not applicable.

\section{Competing interests}

The authors declare that they have no competing interests.

\section{Author details}

'Department of Obstetrics and Gynecology, The affiliated Obstetrics and Gynecology Hospital with Nanjing Medical University; Nanjing Maternity and Child Health Care Hospital, 123 Tianfeixiang, Mochou Road, Qinhuai District, Nanjing 210004, China. ${ }^{2}$ Department of Clinical Nutrition, Nanjing University Medical School Affiliated Nanjing Drum Tower Hospital, 321 Zhongshan Road, Gulou District, Nanjing, China. ${ }^{3}$ Nanjing Maternal and Child Health Institute, 123 Tianfeixiang, Mochou Road, Qinhuai District, Nanjing, China. ${ }^{4}$ Department of Science and Education, The affiliated Obstetrics and Gynecology Hospital with Nanjing Medical University; Nanjing Maternity and Child Health Care Hospital, 123 Tianfeixiang, Mochou Road, Qinhuai District, Nanjing, China. ${ }^{5}$ Department of Medical Records, The affiliated Obstetrics and Gynecology Hospital with Nanjing Medical University; Nanjing Maternity and Child Health Care Hospital, 123 Tianfeixiang, Mochou Road, Qinhuai District, Nanjing, China. ${ }^{6}$ Department of Prenatal Diagnosis, Women's Hospital of Nanjing Medical University, Nanjing Maternity and Child Health Care Hospital, 123 Tianfeixiang, Mochou Road, Qinhuai District, Nanjing, China. 
Received: 17 June 2019 Accepted: 14 October 2019

Published online: 01 November 2019

\section{References}

1. Medici M, Korevaar T, Schalekamp-Timmermans S, Gaillard R, de Rijke YB, Visser WE, et al. Maternal early-pregnancy thyroid function is associated with subsequent hypertensive disorders of pregnancy: the generation $\mathrm{R}$ study. J Clin Endocrinol Metab. 2014;99:E2591-8.

2. Steegers EA, von Dadelszen P, Duvekot JJ, Pijnenborg R. Pre-eclampsia. Lancet. 2010;376:631-44.

3. Armaly Z, Jadaon JE, Jabbour A, Abassi ZA. Preeclampsia: Novel Mechanisms and Potential Therapeutic Approaches. Front Physiol. 2018;9:973.

4. Chen J, Khalil RA. Matrix Metalloproteinases in Normal Pregnancy and Preeclampsia. Prog Mol Biol Transl Sci. 2017;148:87-165.

5. Hod T, Ana Sofia Cerdeira S, Karumanchi A. Molecular Mechanisms of Preeclampsia. Cold Spring Harb Perspect Med. 2015;5:a023473.

6. Possomato-Vieira JS, Khalil RA. Mechanisms of Endothelial Dysfunction in Hypertensive Pregnancy and Preeclampsia. Adv Pharmacol. 2016;77:361-431.

7. Ilekis JV, Tsilou E, Fisher S, Abrahams VM, Soares MJ, Cross JC, et al. Placental Origins of Adverse Pregnancy Outcomes: Potential Molecular Targets- An Executive Workshop Summary of the Eunice Kennedy Shriver National Institute of Child Health and Human Development. Am J Obstet Gynecol. 2016;215:S1-S46.

8. Spradley FT, Palei AC, Granger JP. Increased risk for the development of preeclampsia in obese pregnancies: weighing in on the mechanisms. Am J Physiol Regul Integr Comp Physiol. 2015;309:R1326-43.

9. Barjaktarovic M, Korevaar TIM, Chaker L, Jaddoe WW, de Rijke YB, Visser TJ, et al. The association of maternal thyroid function with placental hemodynamics. Hum Reprod. 2017;32:653-61.

10. Bamfo JE, Kametas NA, Chambers JB, Nicolaides KH. Maternal cardiac function in normotensive and pre-eclamptic intrauterine growth restriction. Ultrasound Obstet Gynecol. 2008;32:682-6.

11. Prefumo F, Muiesan ML, Perini R, Paini A, Bonzi B, Lojacono A, Agabiti-Rosei E, Frusca T. Maternal cardiovascular function in pregnancies complicated by intrauterine growth restriction. Ultrasound Obstet Gynecol. 2008;31:65-71.

12. Valensise H, Novelli GP, Vasapollo B, Borzi M, Arduini D, Galante A, Romanini C. Maternal cardiac systolic and diastolic function: relationship with uteroplacental resistances. A Doppler and echocardiographic longitudinal study. Ultrasound Obstet Gynecol. 2000;15:487-97.

13. Ghi T, Kulevak M, Youssef A, Maroni E, Nanni M, Pilu G, Rizzo N, Pelusi G. Maternal cardiac function in complicated twin pregnancy: a longitudinal study. Ultrasound Obstet Gynecol. 2011;38:581-5.

14. Tay J, Lin F, Masini G, Bennett PR, McEniery CM, lan B. Wilkinson, et al. Early and late preeclampsia are characterized by high cardiac output, but in the presence of fetal growth restriction, cardiac output is low: insights from a prospective study. Am J Obstet Gynecol. 2018;S0002937818306501.

15. Wilson KL, Casey BM, Mclntire DD, Halvorson LM, Cunningham FG. Subclinical thyroid disease and the incidence of hypertension in pregnancy. Obstet Gynecol. 2012;119:315-20.

16. Aggarawal N, Suri V, Singla R, Chopra S, Sikka P, Shah VN, Bhansali A. Pregnancy outcome in hyperthyroidism: a case control study. Gynecol Obstet Investig. 2014;77:94-9.

17. Vasilopoulou E, Loubiere LS, Lash GE, Ohizua O, McCabe CJ, Franklyn JA, Killby MD, Chan SY. Triiodothyronine regulates angiogenic growth factor and cytokine secretion by isolated human decidual cells in a cell-type specific and gestational age-dependent manner. Hum Reprod. 2014;29:1161-72.

18. Carneiro-Ramos MS, Diniz GP, Almeida J, Vieira RLP, Pinheiro SVB, Santos RA Barreto-Chaves MLM. Cardiac angiotensin II type I and type II receptors are increased in rats submitted to experimental hypothyroidism. J Physiol. 2007 ; 583:213-23.

19. Brown MA, Lindheimer MD, de Swiet M, Van Assche A, Moutquin JM. The classification and diagnosis of the hypertensive disorders of pregnancy: statement from the International Society for the Study of Hypertension in Pregnancy (ISSHP). Hypertens Pregnancy. 2001;20:IX-XIV.

20. Mclntyre JPR, Ellyett KM, Mitchell EA, Quill GM, Thompson JMD, Stewart AW, et al. Validation of thoracic impedance cardiography by echocardiography in healthy late pregnancy. BMC Pregnancy Childbirth. 2015;15:70.

21. Lazarus J, Brown RS, Daumerie C, Hubalewska-Dydejczyk A, Negro R, Vaidya B. 2014 European thyroid association guidelines for the Management of Subclinical Hypothyroidism in pregnancy and in children. Eur Thyroid J. 2014;3:76-94.
22. Liu Y, Chen H, Jing C, Li FP. The Association Between Maternal Subclinical Hypothyroidism and Growth, Development, and Childhood Intelligence: A Meta-analysis. J Clin Res Pediatr Endocrinol. 2018;10:153-61.

23. Al Shangeeti SA, Alkhudairy YN, Alabdulwahed AA, Ahmed AE, Al-Adham MS, Mahmood NM. Prevalence of subclinical hypothyroidism in pregnancy in Saudi Arabia. Saudi Med J. 2018;39:254-60.

24. Duncan Bassett JH, Williams GR. Role of Thyroid Hormones in Skeletal Development and Bone Maintenance. Endocr Rev. 2016;37:135-87.

25. Poppe K, Glinoer D. Thyroid autoimmunity and hypothyroidism before and during pregnancy. Hum Reprod Update. 2003;9:149-61.

26. Yu N, Cheng YJ, Liu LJ, Sara JDS, Cao ZY, Zheng WP, et al. What is the association of hypothyroidism with risks of cardiovascular events and mortality? A meta-analysis of 55 cohort studies involving 1,898,314 participants. BMC Med. 2017;15:21.

27. Shao Q, Cheng HJ, Callahan MF, Kitzman DW, Li WM, Cheng CP. Overexpression myocardial inducible nitric oxide synthase exacerbates cardiac dysfunction and beta-adrenergic desensitization in experimental hypothyroidism. Int J Cardiol. 2016;204:229-41.

28. Li X, Yao Y, Chen Z, Fan S, Hua W, Zhang S, et al. Thyroid-stimulating hormone within the normal range and risk of major adverse cardiovascular events in nonischemic dilated cardiomyopathy patients with severe left ventricular dysfunction. Clin Cardiol. 2019. https://doi.org/10.1002/clc.23117.

29. Biondi B. Mechanisms in endocrinology: heart failure and thyroid dysfunction. Eur J Endocrinol. 2012;167:609-18.

30. Biondi B, Cooper DS. The clinical significance of subclinical thyroid dysfunction. Endocr Rev. 2008;29:76-131.

31. Klein I, Ojamaa K. Thyroid hormone and the cardiovascular system. N Engl J Med. 2001;344:501-9.

32. Danzi S, Klein I. Thyroid hormone and the cardiovascular system. Minerva Endocrinol. 2004;29:139-50.

33. Roef GL, Taes YE, Kaufman J-M, Van Daele CM, De Buyzere ML, Gillebert TC, et al. Thyroid Hormone Levels Within Reference Range Are Associated with Heart Rate, Cardiac Structure, and Function in Middle-Aged Men and Women. Thyroid. 2013;23:947-54.

34. Gao C, Li T, Liu J, Guo Q, Tian L. Endothelial Functioning and Hemodynamic Parameters in Rats with Subclinical Hypothyroid and the Effects of Thyroxine Replacement. PLoS One. 2015;10:e0131776.

35. Öner T, Özdemir R, Doksöz Ö, Yozgat Y, Karadeniz C, Demirpençe S, et al. Cardiac Function in Newborns with Congenital Hypothyroidism: Association with Thyroid-Stimulating Hormone Levels. J Clin Res Pediatr Endocrinol. 2015;7:307-11.

36. John W, Petersen JL, Chi Y-Y, Lingis M, Williams RS, Rhoton-Vlasak A, et al. Comparison of multiple non-invasive methods of measuring cardiac output during pregnancy reveals marked heterogeneity in the magnitude of cardiac output change between women. Physiol Rep. 2017;5:e13223.

37. Andreas M, Kuessel L, Kastl SP, Wirth S, Gruber K, Rhomberg F, et al. Bioimpedance cardiography in pregnancy: A longitudinal cohort study on hemodynamic pattern and outcome. BMC Pregnancy and Childbirth. 2016;16:128.

38. Gabbe S, Niebyl J, Simpson J. Obstetrics: normal and problem pregnancies. Philadelphia: Saunders; 2012. p. 779-824.

39. Lavie A, Ra M, Lev S, Blecher Y, Amikam U, Shulman Y, et al. Maternal cardiovascular hemodynamics in normotensive versus preeclamptic pregnancies: a prospective longitudinal study using a noninvasive cardiac system (NICaS ${ }^{\text {TM }}$ ). BMC Pregnancy and Childbirth. 2018;18:229.

40. Tudoran C, Tudoran M, Vlad M, Balas M, Pop GN, Parv F. Echocardiographic evolution of pulmonary hypertension in female patients with hyperthyroidism. Anatol J Cardiol. 2018;20:174-81.

\section{Publisher's Note}

Springer Nature remains neutral with regard to jurisdictional claims in published maps and institutional affiliations. 